\title{
Experimental Investigation of the Mathematical Model for Optimum Holding Force of a Bernoulli Pad
}

\section{Rujeko Masike ${ }^{1^{*}}$ and Janak Kumar B Patel ${ }^{2}$}

\author{
${ }^{1}$ Department of Industrial and Manufacturing Engineering, Harare Institute of Technology, Zimbabwe \\ ${ }^{2}$ Department of Electronics and Communication Engineering, Amity University, India
}

\begin{abstract}
Holding force for a climbing robot is of paramount importance and has been intensely investigated in the last decade. Emphasis of this project has been on minimizing energy expenditure, increasing payload and traversing different wall materials. To achieve this two important factors have been identified for pneumatics based holding pads, namely pressure and height from surface. This paper presents the characterisation of these parameters that influence holding force in a Bernoulli pad for the purpose of optimising energy expenditure. An experimental identification of a mathematical model for a Bernoulli principle based holding force is described. Force analysis through experimental verification for a commercially available Bernoulli pad model was done, so as to quantitatively evaluate parameters of the pad to achieve attachment on a wall. Factors that influence the holding force, such as air velocity and height of pad from surface were experimentally investigated and their cause and effect established. The cause and effect of these parameters were confirmed through regression analysis using Matlab R2016a. Close correspondence between the experimental and simulated results indicated that the developed model is accurate enough for design and implementation of an optimum force for climbing robot. The methods proposed in this study are valuable in guiding the design of pneumatic based adhesion devices, such as wall-climbing robots.
\end{abstract}

\section{Keywords}

Adhesion, Bernoulli pad, Climbing robot, Pneumatic force

\section{Introduction}

Using the Bernoulli principle as inspiration, important principles are revealed for reliable maneuvering of robotic manipulators on vertical structures. When people grip objects, they change the grip force relative to the object structure $[1,2]$. The same applies to traversing vertical structures with different surfaces and elevations. A variable hold- ing force will be required at any given scenario. Hence this paper describes the development and verification of a proposed mathematical model for an adaptive holding force of a vertical wall climbing robot with Bernoulli pads.

In this paper, a Bernoulli pad model has been adopted because they have high force/weight ratio and good adaptability to various surface

*Corresponding author: Rujeko Masike, Department of Industrial and Manufacturing Engineering, Harare Institute of Technology, Zimbabwe

Accepted: September 16, 2019; Published: September 18, 2019

Copyright: (C) 2019 Masike R, et al. This is an open-access article distributed under the terms of the Creative Commons Attribution License, which permits unrestricted use, distribution, and reproduction in any medium, provided the original author and source are credited.

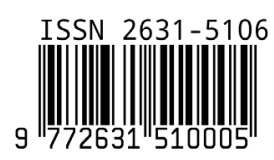

Masike and Patel. Int J Robot Eng 2019, 4:016 
conditions. They use compressed air to provide an adhesion force based on the Bernoulli principle, which creates a low pressure region near the surface $[3,4]$. Hence the Bernoulli principle adhesion mechanism is proposed as being suitable for implementation in a proposed adaptive control scheme. There are examples of climbing robots using Bernoulli principle adhesion mechanism that were modified geometrically to increase the holding force by five times. The researchers added a pintle to the design which introduced aerodynamic effects thereby increasing the holding force [5]. Other researchers have looked at varying the controllable factors of the Bernoulli principle like air pressure and height from surface $[6,7]$. However these are not holistic approaches in that they look at only one parameter and do not account simultaneously for the other controllable parameters which affect optimal holding force with manual regulating mechanisms. The work will lead to the determination of optimal holding force for the pad, and achieve low air consumption, by experimentally specifying the parameters affecting holding force in a Bernoulli pad and creating a mathematical model of the relationship amongst parameters. With this, the researchers will finally create an adaptive control for the pad to operate in different environments, ensuring low self-weight, high payload, and reduced energy consumption.

\section{Holding force}

While the Bernoulli equation is stated in terms of universally valid ideals like conservation of energy in terms of pressure, kinetic energy and potential energy, its application is limited to cases of steady flow. If the flow is to a good approximation laminar, then the kinetic energy of flow at any point of the fluid can be modelled and calculated with the assumption that all the kinetic energy of the fluid is contributing directly to the forward flow process of the fluid [8] giving:

$$
\begin{aligned}
& \frac{P_{1}}{\rho g}+\frac{v_{1}^{2}}{2 g}+h_{1}=\frac{P_{2}}{\rho g}+\frac{v_{2}^{2}}{2 g}+h_{2} \\
& \text { If } h_{1}=h_{2} \text { then, } \\
& P+\frac{1}{2} \rho V^{2}+\rho g h=\text { constant }
\end{aligned}
$$

Where $P=$ pressure, $\rho=$ density, $V=$ velocity, $g$ $=$ gravitational acceleration, $h=$ height.

While conservation of energy always applies,

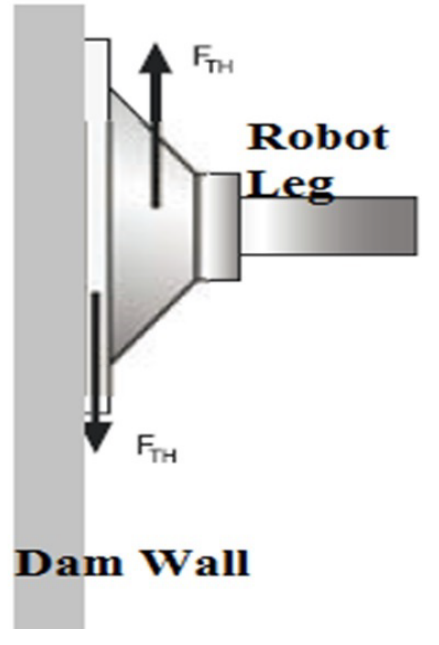

Figure 1: Holding force scenario on dam wall.

the Bernoulli equation certainly does not describe how that energy is distributed under transient conditions. In spite of these restrictions, the equation is very useful, because it can give great insight into the balance between pressure, height, surface conditions and robot state. This paper presents experimentation of the actual flow of energy in a Bernoulli pad and how it affects the holding force of the pad.

Equation 3 and Figure 1, show the parameters which affect holding force for the Bernoulli pad. Hence:

$$
F_{T H}=\left(\frac{m}{\mu}\right) \times(g+a) \times S
$$

Where: $F_{T H}=$ theoretical holding force, $\mathrm{m}=$ mass $/ \mathrm{kg}, \mathrm{g}=$ gravitational acceleration $\mathrm{m} / \mathrm{s}^{2}, \mathrm{a}=$ system acceleration $\mathrm{m} / \mathrm{s}^{2}, \mu=$ coefficient of friction, $\mathrm{S}=$ safety factor (min value 2 ).

Hence combining the Bernoulli equation (2) and Theoretical holding force equations (3) gives:

$$
P+\rho \frac{v^{2}}{2}+\rho g h=\frac{m}{\mu} x(g+a) x S
$$

Equation (4) illustrates that when the object is stationary then $a=0$ therefore the holding force largely depends on $\mu$ which is the roughness of the terrain. Hence the proposed pad will adjust holding force in relation to the frictional force of the surface and the Bernoulli parameters in the pad determined experimentally. The intention is to develop a system that automatically adjust holding force in relation to $\mu, \mathrm{P}, \mathrm{h}$ and a, through development of an algorithm which searches 


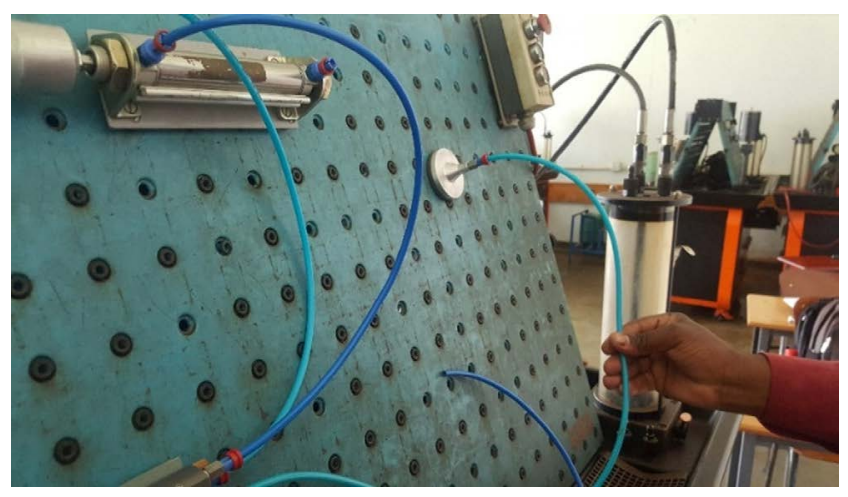

Figure 2: A single Bernoulli pad mounted with pneumatic line.

for an optimum holding force with respect to the surface and robot state in response to given sensor data.

\section{Materials and Methods}

The controllable input factors include height of pad from surface and pressure; and uncontrollable factors include surface condition and robot state. The controllable input factors can be modified to optimize the output which is holding force. Hence the experiments carried out aimed to determine the controllable factors in the Bernoulli pad, how they interact and influence each other so they can then be modelled into a mathematical form for optimisation in a dynamic environment behavior. This will eventually lead to the development of an adaptive climbing mechanism. The difference between the desired holding force and the behavior of the system will determine variation of parameters hence maintaining optimal holding force.

\section{Pressure versus force}

A model of a Rexroth type pad was used, Figure 2 , which is $60 \mathrm{~mm}$ diameter and weight $130 \mathrm{~g}$. A $3 / 2$ way pneumatic valve, standard nominal flow rate 100 liters per min and pressure operating range 0-100 bars with nozzle size $4 \mathrm{~mm}$ was used for the experiments. The weights lifted by the pad ranged from a paper weighing $5.8 \mathrm{~g}$ to a sheet metal weighing $200 \mathrm{~g}$. In the lab these represent different force requirements for different surfaces. The following tools were used:

- Predesigned robot for measuring force requirements

- MATLAB 2016 software to simulate and model the pad

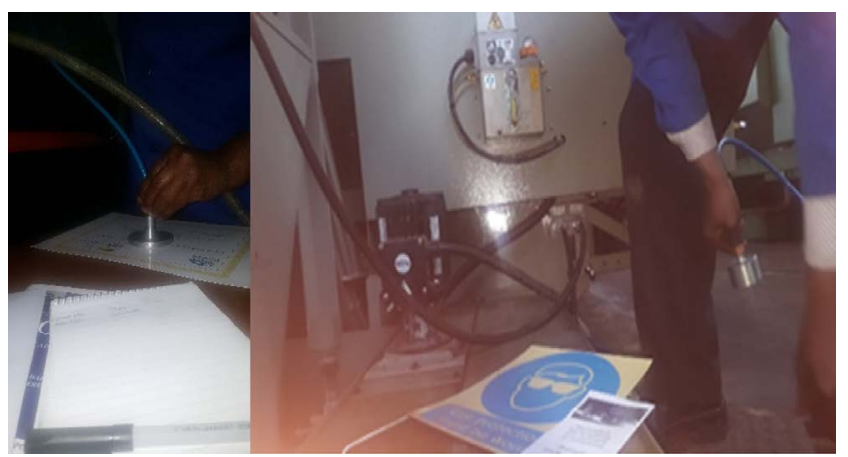

Figure 3: Pressure and height requirements determination.

Eventually the results from these experiments will then be extrapolated to a dam wall characteristics for optimal holding force on the vertical surface. The aim of this experiment was to determine the effect on force of increasing the pressure on a Bernoulli gripper. Different air pressures were applied and different weights lifted to determine the pressure requirements. This is a simplified set up for laboratory purposes to get the relationship between pressure and force (mg). Figure 3 shows the experimental setup.

\section{Height versus force}

The lifting force was measured with the help of a tensile strength tester (type Zwick 1484) with a movable test bed and a fixed force sensor at the top. The force sensor had a range of $5 \mathrm{~N}$ and nominal sensitivity of $2 \mathrm{mV} / \mathrm{V}$ (type HBM-U1). The pad was attached to the force sensor. The object was attached to the test bed surface to be able to register the generated lifting force.

\section{Results}

It was seen that increasing the pressure supplied, results in an increase in lifting force until the maximum is reached. After reaching the maximum the object releases partly until complete release as shown in Figure 4. The reason for this decrease in force could be that for Bernoulli's principle, the fluid should be incompressible. However, air is compressible and the limit of the flow velocity for compressible fluids is until $100 \mathrm{~m} / \mathrm{s}$ [9-16]. This could explain the decrease in lifting force. Hence a model which keeps the pressure within range is needed.

The lifting force increases with height from object until a certain distance where it starts decreasing as shown in Figure 5. In the robot Bernoulli pad we need to keep holding force optimal therefore 


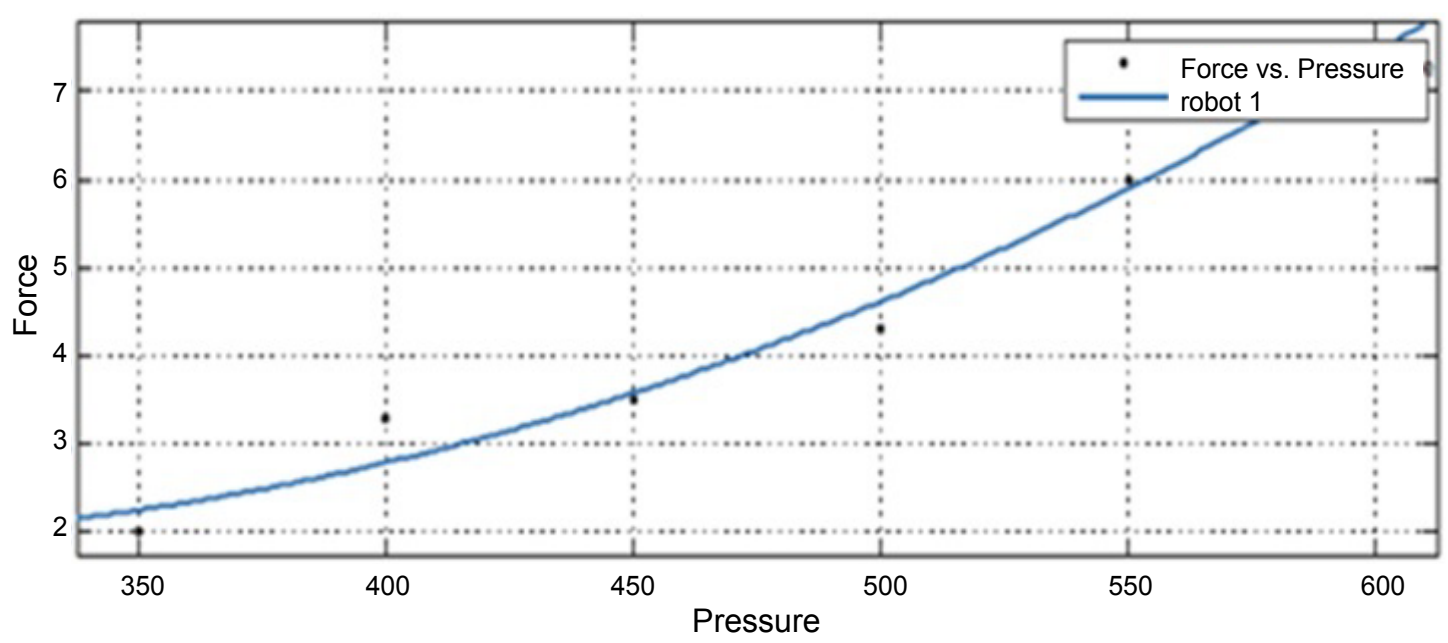

Figure 4: Pressure-force relationship.

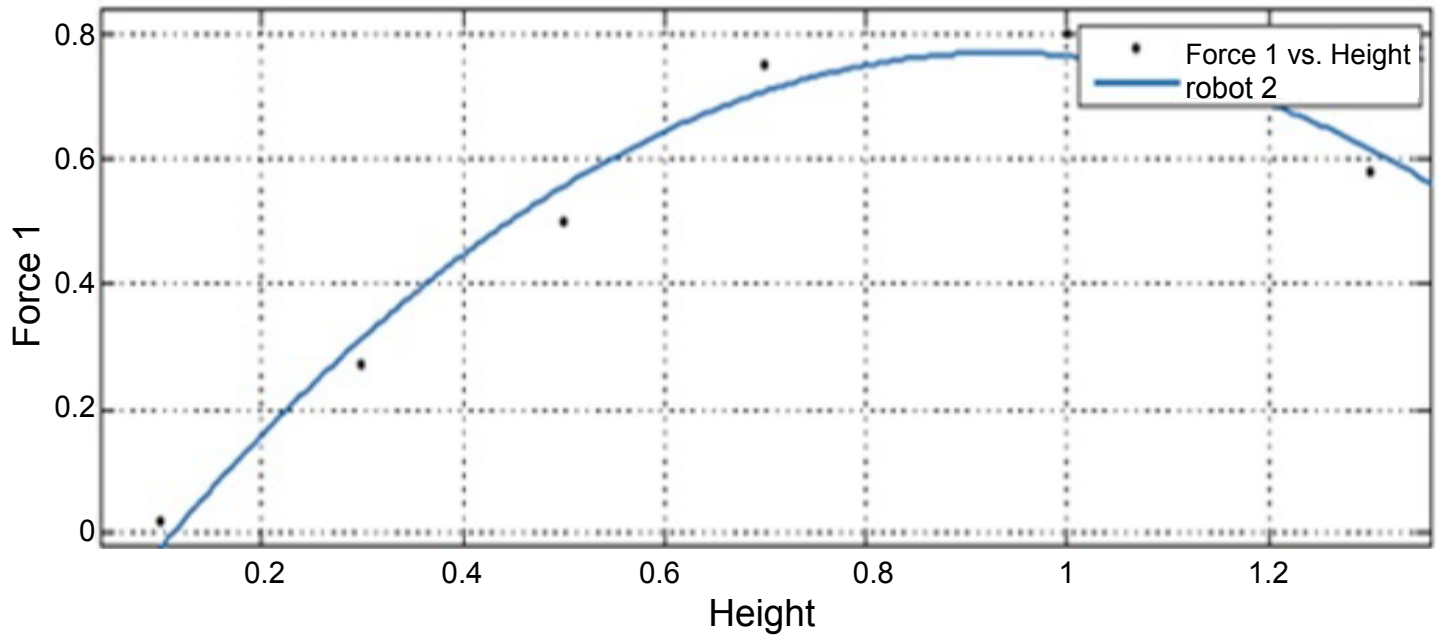

Figure 5: Height-force relationship.

there is need to control height in the dynamic environment.

\section{Mathematical model}

In the mathematical model development $x=h$, and $y=P$.

The results obtained in the above experiments were entered in MATLAB 2016a and extrapolated to give the following equations, with varying polynomials of $x$ and $y$, and coefficients with $95 \%$ confidence bounds:

Linear model Polynomial 22:

$f(x, y)=-9.695+21.41 x-1.588 y-10.41 x^{2}+0.4529 x y$ $+0.6011 y^{2}$

Goodness of fit:
Standard Deviation (SSE) $=2.041 e^{-29}$, RootSquare (R-square) $=1$, Adjusted R-square: $\mathrm{NaN}$, Root Mean Square Error (RMSE): NaN

Linear model Polynomial 12:

$$
f(x, y)=-0.607+1.199 x+1.166 y-1.511 x+
$$$$
0.4114 y^{2}
$$

Goodness of fit:

SSE $=0.01016$, R-square $=0.942$, Adjusted R-square $=0.7098$, RMSE $=0.1008$

Linear model Polynomial 11:

$$
f(x, y)=0.6622-0.6172 x+0.6021 y
$$

Goodness of fit:

SSE $=0.01346$, R-square $=0.9231$, Adjusted R-square $=0.8719$, RMSE $=0.06697$ 
Linear model Polynomial 21:

$f(x, y)=-7.219+16.8 x+16.0 y-9.068 x^{2}+1.846 x y(8)$

Goodness of fit:

SSE $=0.004143$, R-square $=0.9763$, Adjusted R-square $=0.8816$, RMSE $=0.06437$

From these results, equation (5) has the closest fit since RMS is 1 . It means all the values can be explained by the equation with minimum error. It is the best representative of the relationship between holding force, pressure and height.

Hence simplifying equation (4) gives:

$$
P+h=\frac{m s}{\mu \rho}+\frac{m s a}{\mu \rho g}-\frac{v^{2}}{2 g}
$$

Substituting $P+h$ with equation (5) gives us: $-9.695+21.41 x-1.588 y-10.41 x^{2}+0.4529 x y+0.6011 y^{2}=\frac{m s}{\mu \rho}+\frac{m s a}{\mu \rho g}-\frac{v^{2}}{2 g}$

Equation (10) then gives us the relationship between variable parameters and fixed parameters affecting holding force in a pad. Hence algorithms for adaptive control can now be sought. This is the next part of the project.

\section{Validation of results}

An already existing climbing robot using suction pads was used to determine the force requirements on different surfaces. The Bernoulli pad model was tested in real time wall surface environments. Table 1 represents the comparison between actual force requirements for an already existing robot and force produced for the Bernoulli pad model. This investigation was carried out to find the adherence characteristics of the Bernoulli pad to different wall surfaces and helps to find the holding capacity of the pad. The experimental analysis is carried out with the prototype shown in Figure 6.

\section{Discussion}

Climbing robots make use of the different ad-

Table 1: Comparison of actual versus experimental force.

\begin{tabular}{|l|l|l|l|}
\hline Weight/g & $\begin{array}{l}\text { Force } \\
\text { requirement/N }\end{array}$ & $\begin{array}{l}\text { Experimental } \\
\text { Force/N }\end{array}$ & Variation \\
\hline 5.8 & 0.056 & 0.40 & 0.344 \\
\hline 20 & 0.196 & 0.50 & 0.304 \\
\hline 55 & 0.541 & 0.69 & 0.149 \\
\hline 105 & 1.03 & 0.59 & -0.44 \\
\hline 200 & 1.962 & 0.89 & -1.072 \\
\hline
\end{tabular}

hesion systems available, and there are examples of robots that can perform a search to obtain a secure placement of end effector. However, there are currently none that use the attachment mechanism in an energy efficient manner [4]. Albagul, et al. proposed suction as means of sticking to the wall. However this work needs proper design to make sure that all of sucking cups are vertically oriented to the wall surface during the rotational motion of the legs, and using vacuum pumps of high vacuum level application so that the suction cups adhere on the surface of ordinary wall building [17]. This has the effect of high pressure hence high energy requirements on the robot design. Kishan Panchal, et al. proposed the centrifugal impeller means of adhesion [18]. No perfect sealing is required which is the main advantage of this method. However, they just took the baseline equations as they are and did not explore exactly the interrelations of parameters within the impeller. This also leads to the problem of high energy requirements of the robot due to many assumptions. G Muthukumaran, et al. proposed the design of a vacuum generator which is externally positioned and suction is derived through pneumatic supply hose. The robot climbs at a speed of $1.5 \mathrm{~cm} / \mathrm{s}$ [19]. This design also has high energy requirements. In all the above three designs there is no regulation of the air flow and this impacts on the speed and payload of the robots. When the

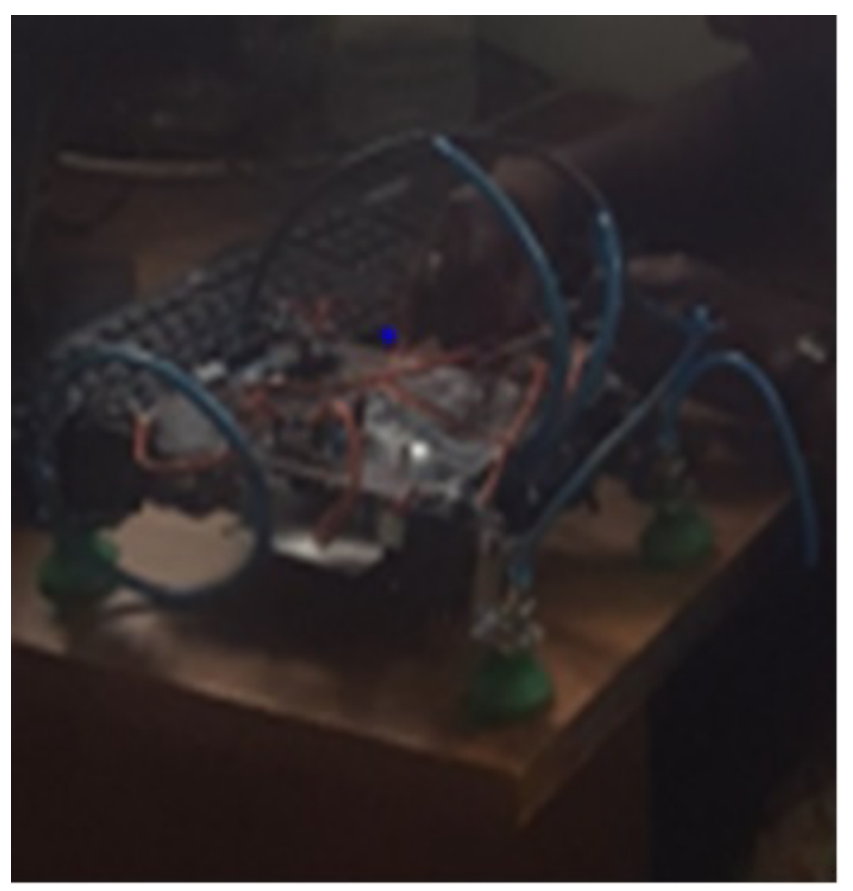

Figure 6: Existing suction cup climbing robot. 


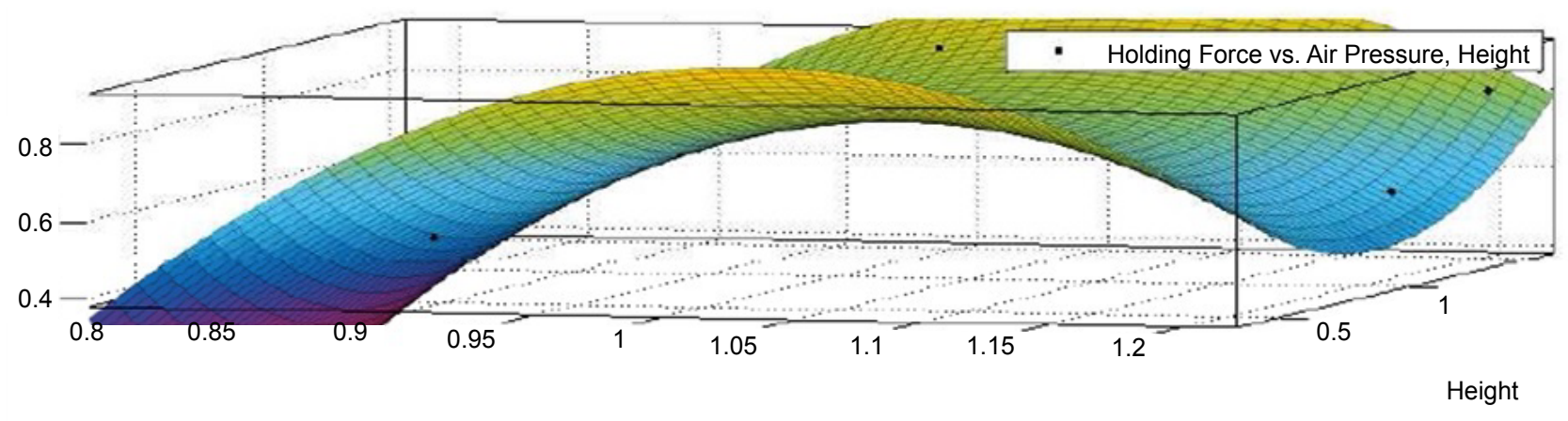

Air Pressure

Figure 7: Pressure/height-force: Depression showing low holding force.

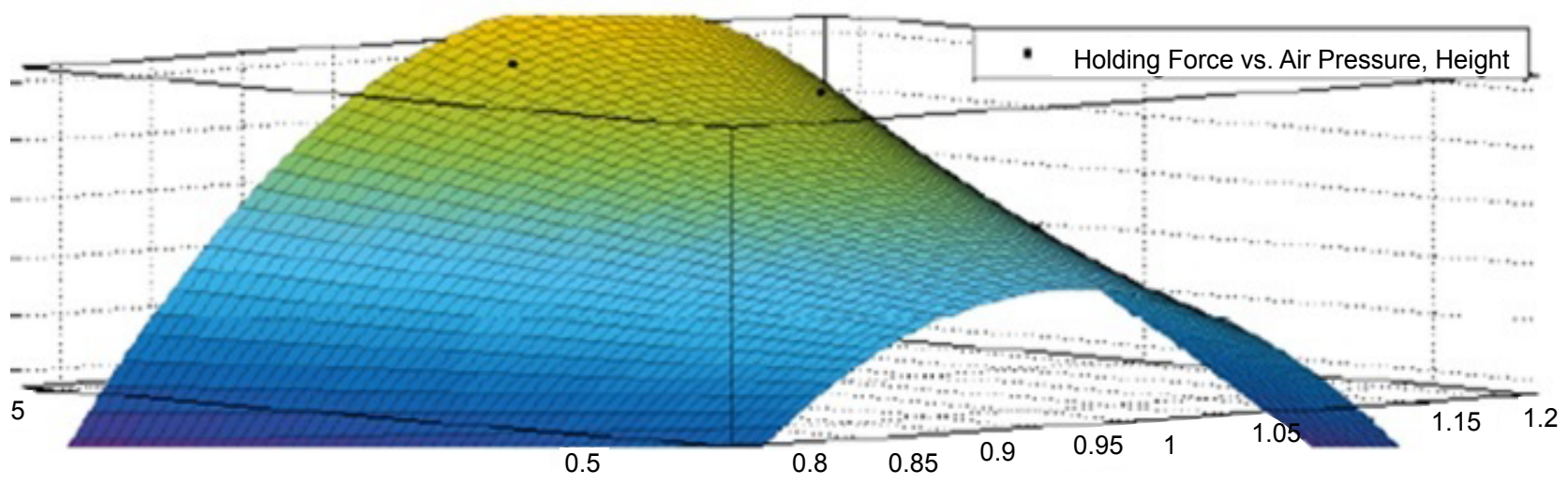

Height

Air Pressure

Figure 8: Pressure/height-force.

robot is climbing the vertical dam wall, the holding force required changes due to the dynamic nature of the environment. This force is determined by the air pressure and height from surface simultaneously, hence the relationship between these two must be determined. MATLAB was used to simulate the experimental results and to extrapolate a mathematical model for this relationship so that it can be controlled. Figure 7 and Figure 8 give schematics of the relationship and it can be seen that a maximum holding force can be reached at certain values of pressure and height. Hence there is need to determine the relationship which gives an optimal force considering the pressure and the height.

Figure 7 has a depression showing low holding force. This is probably conditions which would lead to the robot falling off the wall. Also Table 1 shows that the more the weight increased (Holding Force Requirement) the more the variation increased negatively. Hence there is a limit to the holding force the pad can produce using the proposed mathematical model. This could be due to the assumptions in the Bernoulli equation. The main objective of the proposed final robot is to develop a climbing robot which can adhere to different dam wall conditions and also able to move without falling at any instance. Hence the proposed mathematical model gives adaptability which will then make the robot be able to adjust holding force as per need.

When climbing vertical surfaces the conditions of the surface differ. If the holding force is kept constant, this will result in unnecessary air being used and a cumbersome robot with less carrying ability. Hence by characterizing the parameters affecting holding force, it can be made adaptive therefore reducing on energy requirements and increasing payload.

\section{Conclusion}

The main objective of this paper was to analyse the root parameters leading to an optimum hold- 
ing force in a Bernoulli type pad, and to develop the interrelations between these so as to minimize energy requirements of the pad. The experimental analysis showed that pressure and height from the wall have an effect on the holding force amongst robot state and surface conditions. The mathematical model should be able to give the process dynamics at any given time and enable to adjust the pressure and height in response to the robot state and surface conditions. A comparison of the mathematical model and an existing suction cup climbing robot shows that the model can achieve optimum holding force within certain limits. Hence energy consumption of the Bernoulli pad can be reduced significantly by adopting this mathematical model for adaptive control. The significance of this study is to ultimately have a reliable wall climbing system that can handle different frictional coefficients, avoid falls and increase payload. The future work will involve developing the control algorithm for efficient operation using Artificial Intelligence, and to develop the adaptive control system.

\section{Acknowledgment}

This work was supported in part by the Harare Institute of Technology Research Grant.

Preliminary version of the paper was presented at Joint Event on $5^{\text {th }}$ International Conference on Artificial Intelligence $\& 5^{\text {th }}$ International Conference on Automation \& Robotics ScientificTracks Abstracts: Adv Robot Autom, DOI: 10.4172/21689695-C1-014.

Dr. Eng. Talon Garikayi Director Technology Transfer, Licensing and Commercialisation Centre with the Harare Institute of Technology have provided very valuable guidance and supervision in the writing of this paper.

\section{References}

1. Slota GP, Latash ML, Zatsiorsky VM (2013) Grip forces during object manipulation : Experiment, mathematical model and validation. Exp Brain Res 213: 125-139.

2. https://www.isixsigma.com/tools-templates/ design-of-experiments-doe/design-experiments-E2\%90\%93-primer/

3. www.boschrexroth.com/pneumatics

4. Jacobs SA, Deghani-Sanij AA (2008) Advances in mobile robotics, $11^{\text {th }}$ Int conf on climbing and walking robots and the support technologies for mobile machines, Portugal, 114-121.
5. Journee $M$, Chen X (2011) An investigation into improved non-contact adhesion mechanism for wall climbing robot applications. IEEE International Conference on Robotics and Automation.

6. Davis S, Gray JO, Caldwell DG (2008) An end effector based on the Bernoulli principle for handling sliced fruit and vegetables. Robotics and Computer-Integrated Manufacturing 24: 249-257.

7. Schimdt D, Berns K (2013) Development and applications of a simulation framework for a wall-climbing robot. Intelligent Robots and Systems (IROS), 2013 IEEE/RSJ International Conference, Japan.

8. Dong W, Wang H, Li Z, Jiang Y, Xiao J (2013) Development of a wall-climbing robot with biped-wheel hybrid locomotion mechanism. Intelligent Robots and Systems (IROS), 2013 IEEE/RSJ International Conference, Japan.

9. https://www.destaco.com/end-effectors.html

10.Robosoft (2011) Glass roof cleaning-customised automatic glass-roof cleaning robots.

11.Oliveira ALC, Silva MF, Barbosa RS (2010) Architecture of an wheeled climbing robot with dynamic adjustment of the adhesion system. In: Proceedings of the $8^{\text {th }}$ IEEE International Symposium, Intelligent Systems and Informatics (SISY '10), Subotica, Serbia, 127-132.

12. Rosa GL, Sinatra R (2003) Design of an end-effector of a climbing robot for vertical surfaces. In: Muscato $G$, Longo $D$, Proceedings of the $6^{\text {th }}$ International Conference on Climbing and Walking Robots and the Support Technologies for Mobile Machines (CLAWAR '03), Catania, Italy, 793-800.

13.Cui G, Liang K, Guo J, Li H, Gu D (2012) Design of a climbing robot based on electrically controllable adhesion technology. Int Conf on Solid State and Materials 22 .

14.Armada MA, de Santos PG (2011) Perspectives of climbing and walking robots for the construction industry. In: Berns K, Dillmann R, Proceedings of the $4^{\text {th }}$ International Conference on Climbing and Walking Robots and the Support Technologies for Mobile Machines (CLAWAR '01), Professional Engineering, Karlsruhe, Germany, 929-936.

15.Albitar H, Dandan K, Ananiev A, Kalaykov I (2016) Underwater robotics: Surface cleaning techniques, adhesion and locomotion systems. International Journal of Advanced Robotic Systems 13: 1-14.

16.Silva MF, Tenreiro Machado JA (2008) A survey of technologies and applications for climbing robots locomotion and adhesion. Climbing Robots 1-24. 
17.Albagul A, Asseni A, Khalifa O (2011) Wall climbing robot: Mechanical design and implementation. Recent Advances in Circuits, Systems, Signal and Telecommunications, 28-32.

18. Panchal K, Vyas C, Patel D (2014) Developing the prototype of wall climbing robot. International Journal of Advance Engineering and Research Development 1: 1-5.

19. Muthukumaran G, Ramachandraiah U (2016) Modelling and realization of pneumatics based wall climbing robot for inspection applications. International Journal of Engineering and Technology 8: 1999-2007. 